\title{
Factors mediating the transdifferentiation of islets of Langerhans to duct epithelial-like structures
}

\author{
R Wang, J Li and L Rosenberg \\ Department of Surgery, McGill University, and Research Institute of the McGill University Health Center, Montreal, Quebec, Canada \\ (Requests for offprints should be addressed to L Rosenberg, Department of Surgery, Montreal General Hospital, 1650 Cedar Avenue, Room L9-424, Montreal, \\ Quebec H3G 1A4, Canada; Email: lawrence.rosenberg@mcgill.ca)
}

\begin{abstract}
We have previously shown that isolated islets embedded in type 1 collagen gel in the presence of a defined medium undergo transdifferentiation within $96 \mathrm{~h}$ to duct epithelial structures. The aim of this study was to identify the factors implicated in this process. Freshly isolated canine islets were embedded in type 1 collagen gel, Matrigel or agarose for up to $120 \mathrm{~h}$ and cultured in (i) Dulbecco's modified Eagle's medium (DMEM)/F12 plus cholera toxin (CT), (ii) medium CMRL1066 plus CT, (iii) CMRL1066 plus forskolin and (iv) CMRL1066 alone. At 16 h, intracellular levels of cAMP ( $\mathrm{fmol} / 10^{3}$ islets) were increased in groups i-iii $(642 \pm 17,338 \pm 48,1128 \pm 221)$ compared with group iv (106 $\pm 19, P<0 \cdot 01)$. Epithelial differentiation correlated with the total amount of intracellular cAMP measured over $120 \mathrm{~h}$. Islet-epithelial transformation during the initial $36 \mathrm{~h}$ was associated with a wave of apoptosis which was followed by a wave of cell proliferation. During
\end{abstract}

epithelial differentiation there was a progressive loss of all islet hormones and the concomitant expression of cytoskeletal proteins characteristic of duct epithelial cells. Islets in collagen and Matrigel demonstrated high rates of epithelial differentiation $(63 \pm 2 \%$ and $71 \pm 4 \%$ respectively) compared with those in agarose gel $(0 \pm 0 \%$, $P<0 \cdot 001)$. Islets suspended in DMEM/F12 plus CT supplemented with soluble laminin or fibronectin did not undergo transformation. Prior incubation of freshly isolated islets with an integrin-binding arginine-glycineaspartate motif-presenting synthetic peptide also reduced islet transformation. These studies confirm the biological potential of islets of Langerhans to differentiate to duct epithelial structures. cAMP-mediated signal transduction and an appropriate integrin-matrix interaction are necessary for this process to proceed.

Journal of Endocrinology (2001) 171, 309-318

\section{Introduction}

The process of islet isolation destroys extracellular matrix and eliminates potentially important cellular interrelationships (Rosenberg 1998). Maintenance of cellular phenotype, however, depends on the extracellular milieu, as well as on cytoplasmic signaling proteins and nuclear transcription factors that interact to keep gene expression stable (Okada 1986, Ekblom \& Timpl 1996). It is perhaps not surprising, therefore, that we have observed (Yuan et al. 1996) that islets maintained in culture can undergo a phenotypic switch to duct-like epithelial structures by a process known as transdifferentiation, a change from one differentiated cell phenotype to another, where change includes morphological and functional phenotypic markers (Okada 1986). To understand why islet cells are replaced by duct-like cells, one need only recall pancreatic morphogenesis, during which an epithelial-mesenchymal interaction is soon followed by the appearance of a multipotential stem cell that differentiates into either an exocrine or endocrine phenotype (Bensley 1911, Golosow \& Grobstein 1962, Orci et al. 1979, Dudek \& Lawrence
1988). During this early stage, the cells of the endocrine pancreas develop from progenitor cells that migrate out from the embryonic ductal epithelium (Githens 1986).

Signal transduction in transdifferentiation has only recently become the subject of study; detailed information is therefore unavailable. cAMP-mediated information flow, however, appears to play an important role (Osaka \& Sabban 1997, Ghee et al. 1998, Yarwood et al. 1998). This is of particular interest, because a rise in cAMP has recently been reported to form part of the effector system controlling apoptosis in pancreatic $\beta$-cells (Loweth et al. 1997), and cell loss is the first stage observed in the transformation of islets to duct epithelial structures (Yuan et al. 1996). Moreover, during this transition, islets are maintained in medium supplemented with cholera toxin (CT), which is known to elevate intracellular cAMP.

While the first stage of islet-to-duct transformation is characterized by a loss of endocrine cell mass, this is followed by a period of robust proliferation of new duct-like cells. In contradistinction to the possible role of cAMP-mediated signal transduction in cell loss, in this second stage cell survival may be promoted by extracellular 

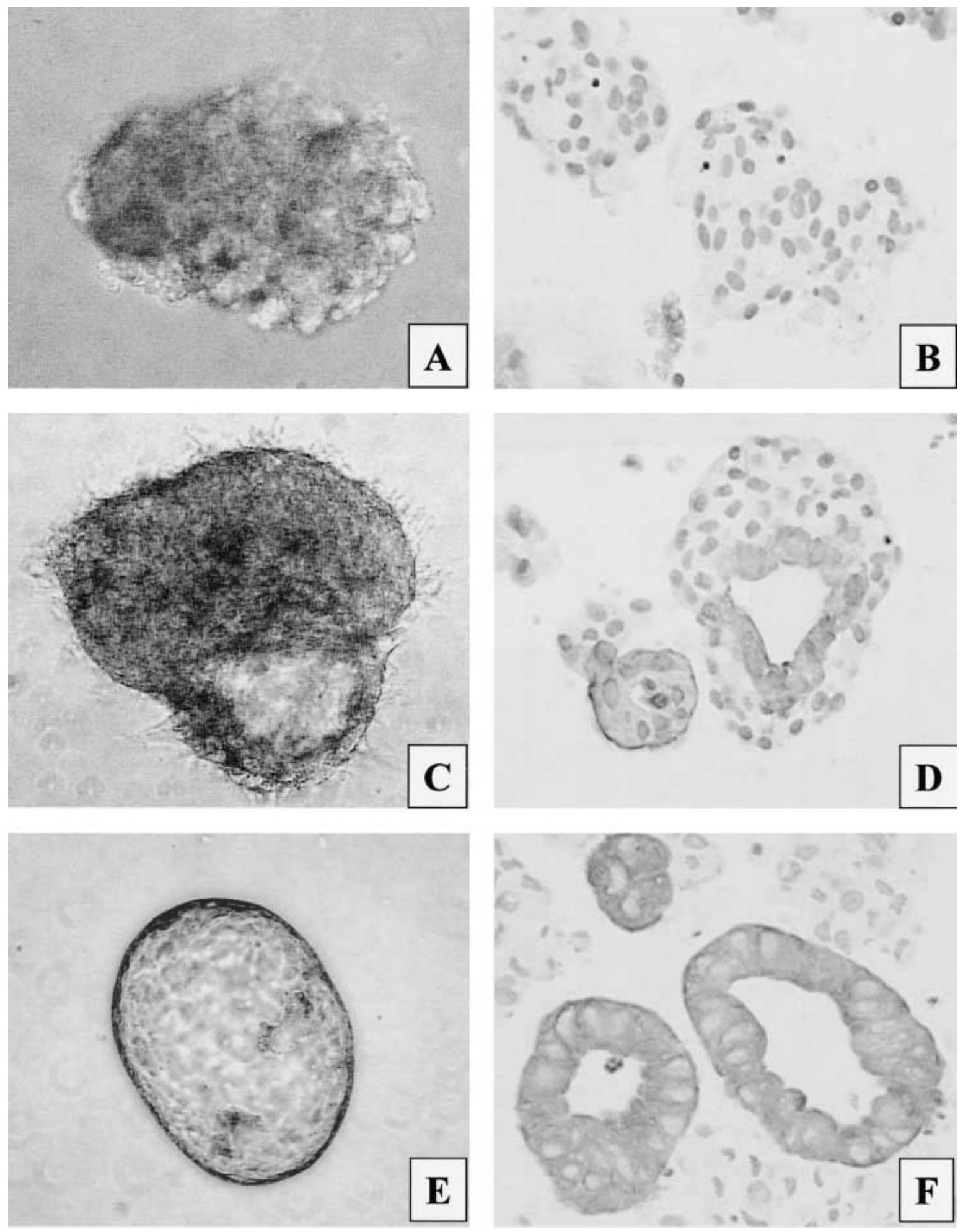

Figure 1 Islets embedded in collagen matrix and maintained in DMEM/F12 plus CT for up to $72 \mathrm{~h}$. The appearance of islets in culture $(A, C$ and $E \times 100)$ and the corresponding light microscopy of tissue sections stained for pan-cytokeratin AE1/AE3 by immunocytochemistry $(\mathrm{B}, \mathrm{D}$ and $\mathrm{F} \times 200)$. 
matrix (Yuan et al. 1996). There is, after all, ample precedent in the literature for cell dependence on extracellular matrix (ECM) for survival (Meredith et al. 1993). This dichotomy of time-dependent signals presents an interesting paradox, the nature of which needs to be further clarified.

The purpose of the present study, therefore, was to characterize the factors involved in the process of islet transdifferentiation to duct epithelial structures, in order to better understand the functional relationships that confer morphogenetic stability on cells in the isolated islet.

\section{Materials and Methods}

\section{Islet isolation and purification}

Pancreata from six mongrel dogs of both sexes (body weight $25-30 \mathrm{~kg}$ ) were resected under general anesthesia in accordance with Canadian Council for Animal Care guidelines (Wang \& Rosenberg 1999). Prior to removal, the pancreatic ducts were cannulated to permit intraductal infusion with Liberase CI $(1.25 \mathrm{mg} / \mathrm{ml}$; Boehringer Mannheim, Indianapolis, IN, USA) according to established protocols (Horaguchi \& Merrell 1981, Ricordi 1992). Purification was achieved by density gradient separation in a three-step EuroFicoll gradient using a COBE 2991 Cell Processor (COBE BCT, Denver, CO, USA) (London et al. 1992). The final preparation consisted of $95 \%$ dithizone-positive structures with diameters ranging from 50 to $500 \mu \mathrm{m}$.

\section{Experimental design}

To evaluate the role of intracellular cAMP, freshly isolated islets were embedded in type 1 collagen gel (Wang \& Rosenberg 1999) and cultured in: (i) Dulbecco's modified Eagle's medium (DMEM)/F12 (GIBCO, Burlington, Ontario, CANADA) supplemented with 10\% fetal bovine serum (FBS), epidermal growth factor (EGF; $100 \mathrm{ng} / \mathrm{ml}$; Sigma, St Louis, MO, USA) and CT (100 ng/ml; Sigma), (ii) medium CMRL1066 (GIBCO) supplemented with $10 \%$ FBS and CT $(100 \mathrm{ng} / \mathrm{ml})$ and $16.5 \mathrm{mM} \mathrm{D}$-glucose, (iii) CMRL1066 supplemented with 10\% FBS and $2 \mu \mathrm{M}$ forskolin (Sigma) and (iv) CMRL1066 supplemented with 10\% FBS. Approximately 3000 islets per group per timepoint were used. Islets were cultured in 95\% air $/ 5 \% \mathrm{CO}_{2}$ at $37^{\circ} \mathrm{C}$, and the medium was changed on alternate days. Representative islets from each group were examined immediately after isolation, and then at 1, 16, 36, 72 and $120 \mathrm{~h}$.

To determine whether the process required a solid gel environment, islets were cultured in suspension in DMEM/F12 with 10\% FBS plus CT and EGF. To determine whether a solid gel environment and extracellular matrix proteins were independent requirements, islets were embedded in 1.5\% agarose gel and maintained in DMEM/F12 with 10\% FBS plus CT and EGF. Alternatively, islets were cultured in suspension in DMEM/F12 with 10\% FBS plus CT and EGF in the presence of soluble laminin $(50 \mu \mathrm{g} / \mathrm{ml})$ or fibronectin ( $50 \mu \mathrm{g} / \mathrm{ml}$; Peninsula Laboratories, Belmont, CA, USA). To determine whether the process was, at least in part, integrin mediated, islets were preincubated at $37^{\circ} \mathrm{C}$ for 60 min either in the presence of the synthetic arginineglycine-aspartate (RGD) motif containing GRGDSP peptide or the control peptide GRGESP $(400 \mu \mathrm{g} / \mathrm{ml}$; Peninsula Laboratories). Finally, to determine whether cystic transformation was dependent on type 1 collagen alone, islets were also embedded in Matrigel (Peninsula Laboratories). The number of islets undergoing cystic transformation were counted under an inverted microscope, and data are reported as the percentage of cystic transformation. For each experiment, values are the average for three flasks and each experiment was repeated six times (i.e. six isolations were performed).

\section{Morphological analysis}

Immunocytochemistry Tissue was fixed in $4 \%$ paraformaldehyde (PFA) and embedded in 2\% agarose following a standard protocol of dehydration and paraffin embedding (Wang \& Rosenberg 1999). A set of six serial sections (thickness $4 \mu \mathrm{m}$ ) was cut from each paraffin block.

Consecutive sections were processed for routine histology and immunostained for pancreatic hormones (insulin, glucagon and somatostatin; Biogenex, San Ramon, CA, USA) and the pan-cytokeratin cocktail AE1/AE3 (Dako, Carpinteria, CA, USA), using the AB complex method (streptavidin-biotin horseradish peroxidase; Dako), as described previously (Wang et al. 1994). For cytokeratin AE1/AE3, sections were pretreated with $0 \cdot 1 \%$ trypsin. The sections were incubated overnight at $4{ }^{\circ} \mathrm{C}$ with the appropriate primary antibodies. Negative controls involved the omission of the primary antibodies.

In situ hybridization In situ hybridization for human proinsulin mRNA (Novocastra, Burlington, Ontario, Canada) was performed on consecutive sections of freshly isolated islets and epithelial cystic structures at $120 \mathrm{~h}$. The sections were hybridized with a fluorescein-labeled oligonucleotide cocktail solution for $2 \mathrm{~h}$ at $37^{\circ} \mathrm{C}$. Slides were then incubated with rabbit Fab anti-FITC (Novocastra), conjugated to alkaline phosphatase antibody (diluted 1:200) for $30 \mathrm{~min}$ at room temperature. The reaction product was visualized by an enzyme-catalyzed color reaction using a nitro blue tetrazolium and $5^{\prime}$-bromo-4chloro-3-indolyl-phosphate kit (Wang et al. 1994, Wang \& Rosenberg 1999).

\section{Analysis of intracellular cAMP level}

Cells were harvested from the collagen gel and washed in $1 \mathrm{mM}$ cold phosphate-buffered saline. Following the 

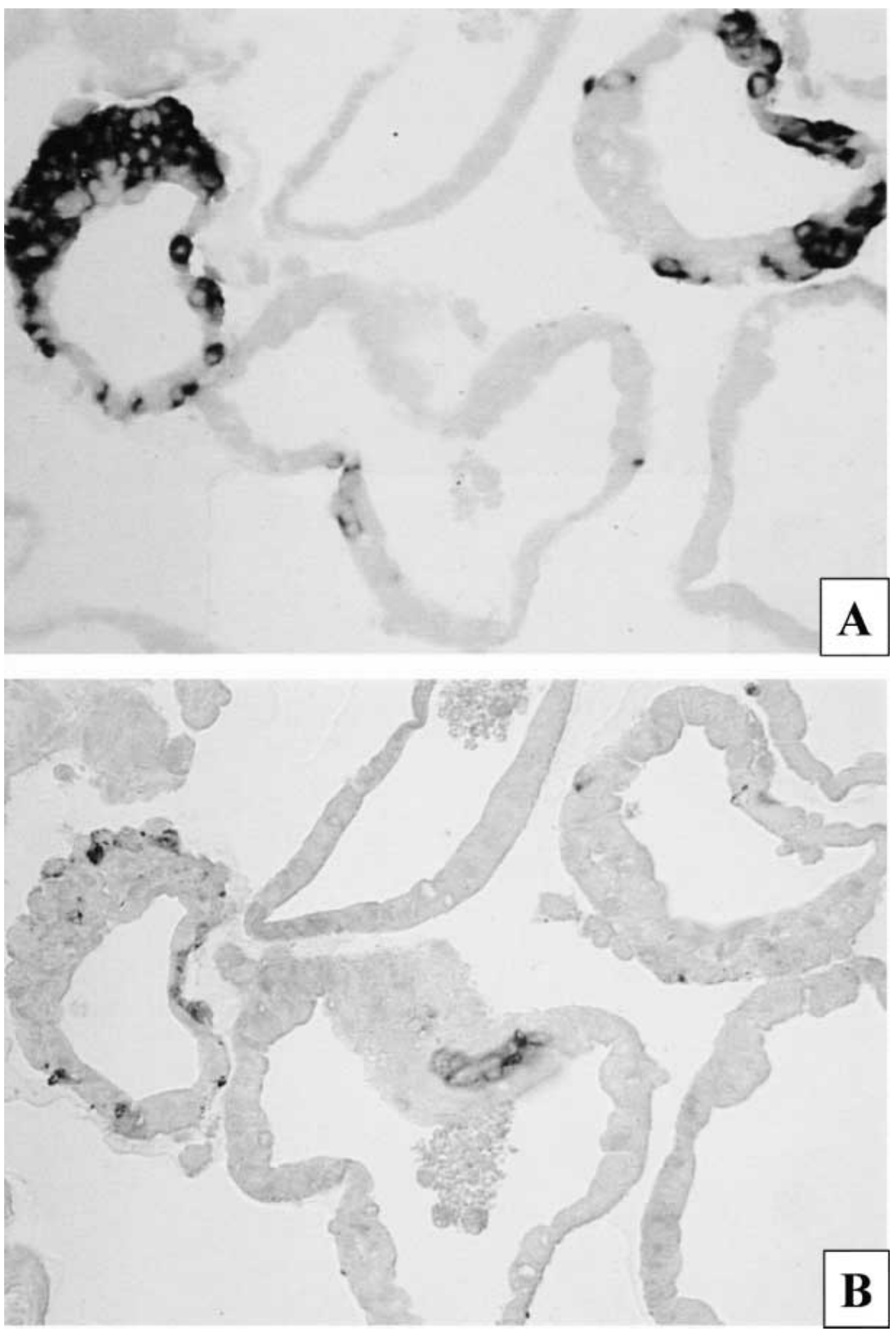

Figure 2 Islets at an intermediate stage of cystic transformation still contain cells that (A) express pro-insulin mRNA, as shown by in situ hybridization, and (B) synthesize and store insulin protein, as shown by immunocytochemistry $\times 400$. 
addition of $200 \mu \mathrm{l}$ lysis buffer, each sample was sonicated for $30 \mathrm{~s}$, then incubated for $5 \mathrm{~min}$ at room temperature. Cell lysate $(100 \mu \mathrm{l})$ was transferred to a donkey anti-rabbit Ig-coated plate. The intracellular cAMP content of nonacetylated samples was measured using a commercially available cAMP enzyme-linked immunoassay (ELISA) kit (assay range 12.5-3200 fmol/well; Amersham International plc, Little Chalfont, Bucks, UK). The analysis was performed for each of six different islet isolations, with three flasks per group per time-point. The data are expressed as fmol $/ 10^{3}$ islets.

\section{Insulin content assay}

Cellular insulin content was measured using a solid-phase radioimmunoassay (Immunocorp, Montreal, Quebec, Canada) (Wang et al. 1999) with a sensitivity of $26.7 \mathrm{pmol} / 1(0.15 \mathrm{ng} / \mathrm{ml})$, an interassay variability of $<5 \%$ and an accuracy of $100 \%$. The kit uses anti-human antibodies that cross-react with canine insulin. Obtained values were corrected for variations in cell number by measuring DNA content using a fluorometric DNA assay (Yuan et al. 1996). The data are expressed as $\mu \mathrm{g} / \mu \mathrm{g}$ DNA.

\section{Cell death and proliferation}

Cells cultured in DMEM/F12 plus CT and CMRL1066 were harvested from the gel using collagenase XI (0.25 mg/ml; Sigma, Montreal, Quebec, Canada) and processed for a specific programmed cell death ELISA which detects histone-associated DNA fragments in the cell cytoplasm - a hallmark of the apoptotic process (Roche Molecular, Montreal, Quebec, Canada) (Paraskevas et al. 2001). Cells were incubated in lysis buffer for $30 \mathrm{~min}$, and the supernatant containing cytoplasmic oligonucleosomes was measured at an absorbance of $405 \mathrm{~nm}$. Variations in sample size were corrected by measuring total sample DNA content (Yuan et al. 1996).

To evaluate cell proliferation, cells cultured in DMEM/ F12-CT and CMRL1066 were preincubated with $10 \mu \mathrm{M}$ 5-bromo-2'-deoxyuridine (BrdU; Sigma) for $1 \mathrm{~h}$ at $37^{\circ} \mathrm{C}$. Harvested cells was fixed in 4\% PFA as described above. Immunostaining for $\mathrm{BrdU}$ was performed using the $\mathrm{AB}$ complex method. The sections were pretreated with $0 \cdot 1 \%$ trypsin and $2 \mathrm{M} \mathrm{HCl}$ denatured DNA. A monoclonal anti-BrdU antibody was used at 1:500 dilution (Sigma).

To calculate a BrdU-labeling index, the number of cells positive for the BrdU reaction was determined and expressed as a percentage of the total number of cells counted. At least 500 cells were counted per section for each experimental group and time-point.

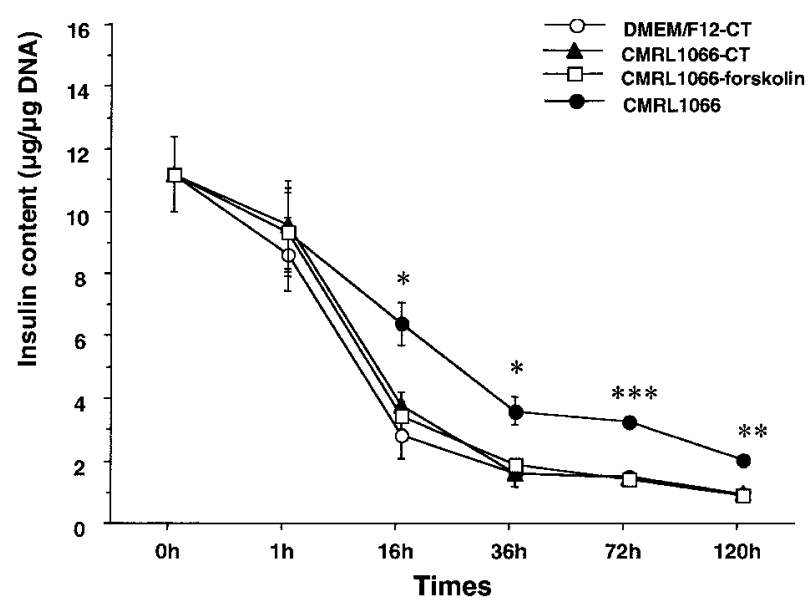

Figure 3 The progressive loss of tissue insulin content during the time-course of cystic transformation. The early rapid decline in insulin content of islets maintained in DMEM/F12 plus CT, CMRL1066 plus CT and CMRL1066 plus forskolin corresponds to the onset of apoptosis by $16 \mathrm{~h}$ (see Fig. 5). Significant differences are shown between treatment with DMEM/F12 plus CT, CMRL1066 plus CT or CMRL1066 plus forskolin vs CMRL1066 alone after 16 h of culture. ${ }^{*} P<0 \cdot 05,{ }^{* *} P<0 \cdot 01,{ }^{* *} P<0 \cdot 001$.

\section{Statistic analysis}

Data obtained from the six different islet isolations are expressed as means \pm s.E.M. The difference between groups was evaluated by one-way analysis of variance.

\section{Results}

\section{Morphological changes}

Under the inverted microscope, freshly isolated islets appeared as solid spheroids. At this time, cytokeratinpositive cells were not demonstrated within islets (Fig. 1A and $\mathrm{B}$ ).

For islets embedded in type 1 collagen and cultured in DMEM/F12 plus CT, CMRL1066 plus CT or CMRL1066 plus forskolin, duct epithelial differentiation was first observed coincident with a loss of cells in the islet periphery, at approximately $16 \mathrm{~h}$. At this time, cells lining the developing cystic spaces were cytokeratin positive (Fig. 1C and D). Fully developed epithelial structures were present in culture by $72 \mathrm{~h}$ (Fig. $1 \mathrm{E}$ and F). Islets cultured in CMRL1066 alone maintained a solid spheroid appearance for the duration of the study and did not undergo epithelial transformation. Immunocytochemical staining did not demonstrate co-localization of cytokeratin and islet cell hormones. This is in keeping with the observation in the intact pancreas that cytokeratin staining was only seen on duct epithelial cells. Pro-insulin gene expression and insulin protein were lost during the period of duct epithelial differentiation (Figs 2 and 3). 

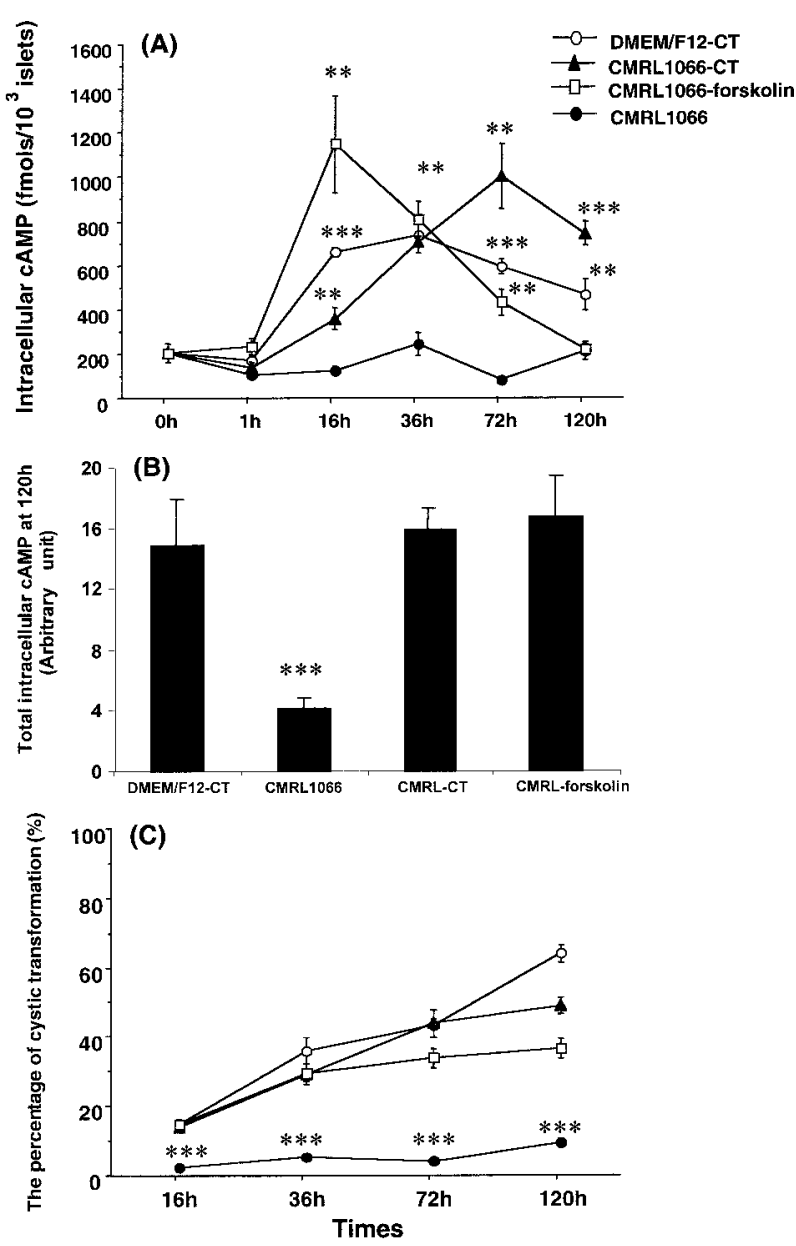

Figure 4 (A) Intracellular levels of cAMP during the time-course of islet-cystic transformation. The means represent the analysis of six different islet isolations, with $n=3$ flasks per group per time-point. (B) The integrated amount of cAMP (area under the curve) measured at $120 \mathrm{~h}$. (C) The percentage of islets undergoing cystic transformation over the time-course of the culture period in the experimental groups. Treatment with DMEM/F12 plus CT, CMRL1066 plus CT or CMRL1066 plus forskolin resulted in significant changes in intracellular cAMP levels compared with CMRL1066 alone after $16 \mathrm{~h}$ of culture. ${ }^{*} P<0 \cdot 05,{ }^{*} P<0 \cdot 01$, ${ }^{* * *} P<0 \cdot 001$.

\section{Intracellular $c A M P$}

After $1 \mathrm{~h}$ intracellular levels of cAMP of islets maintained in DMEM/F12 plus CT, CMRL1066 plus CT and CMRL1066 plus forskolin were significantly elevated compared with freshly isolated islets or to islets maintained in CMRL1066 alone (Fig. 4A). In fact the intracellular level of cAMP of islets cultured in CMRL1066 alone did not increase at all during the time-course of the study. The total intracellular cAMP measured over $120 \mathrm{~h}$ (integrated area under the curve) was similar for islets cultured in DMEM/F12 plus CT, CMRL1066 plus CT and CMRL1066 plus forskolin $(15 \pm 3,16 \pm 2$ and $17 \pm 3$ respectively). In comparison, islets cultured in CMRL1066 alone had the lowest level of total intracellular cAMP $(4 \pm 1, P<0 \cdot 001)$ (Fig. 4B), which was associated with the lowest level of islet-duct transformation (Fig. 4C).

\section{Intracellular insulin content}

The cellular content of insulin (Fig. 3) was highest in freshly isolated islets $(11 \pm 2 \mathrm{ng} / \mu \mathrm{g}$ DNA). Islets maintained in CMRL1066 alone demonstrated a gradual decline in insulin content, in keeping with previously reported observations (Wang \& Rosenberg 1999). In contrast, by $16 \mathrm{~h}$ in culture, the insulin content of cells maintained in DMEM/F12 plus CT, CMRL1066 plus CT and CMRL1066 plus forskolin had declined dramatically, falling to $7 \%$ of the initial value by $120 \mathrm{~h}$, which was approximately $50 \%$ of the intracellular insulin content in islets cultured in CMRL1066 alone $(P<0 \cdot 03$, Fig. 3).

\section{Analysis of cell death and proliferation}

As shown in Fig. 1, cell loss is a feature of islet-to-duct transdifferentiation. However, islet cell apoptosis also occurs naturally when islets are maintained in culture (Wang et al. 1999). Therefore we sought to determine whether cell loss during cystic transformation was associated with an increase in the basal level of programmed cell death. Indeed, using a specific cell death ELISA, we found that a wave of apoptosis occurred sooner $(P<0 \cdot 02)$ and was maintained longer in islets undergoing transdifferentiation in DMEM/F12 plus CT (Fig. 5A), compared with islets maintained under control conditions in CMRL1066 alone. After $36 \mathrm{~h}$, there was no difference between the groups with respect to programmed cell death.

To assess proliferation, cells were labeled with BrdU. Following isolation, the BrdU cell labeling index of islets cultured in DMEM/F12 plus CT was $0 \cdot 8 \%$ - identical to that of islets cultured in CMRL1066 alone. After $36 \mathrm{~h}$, however, a wave of cell proliferation ensued in the DMEM/F12 plus CT group, with the labeling index reaching $18 \%$ at $120 \mathrm{~h}$ (Fig. 5B). In comparison, the labeling index for islets in CMRL1066 remained essentially unchanged throughout the study period $(P<0 \cdot 01)$.

\section{The role of integrin-ECM interactions}

To determine whether elevation of intracellular cAMP was sufficient to induce duct epithelial differentiation, islets were maintained in suspension culture in DMEM/ F12 plusCT and not embedded in collagen gel. Under these conditions, epithelial transformation did not occur. This suggested that an increase in intracellular cAMP was 

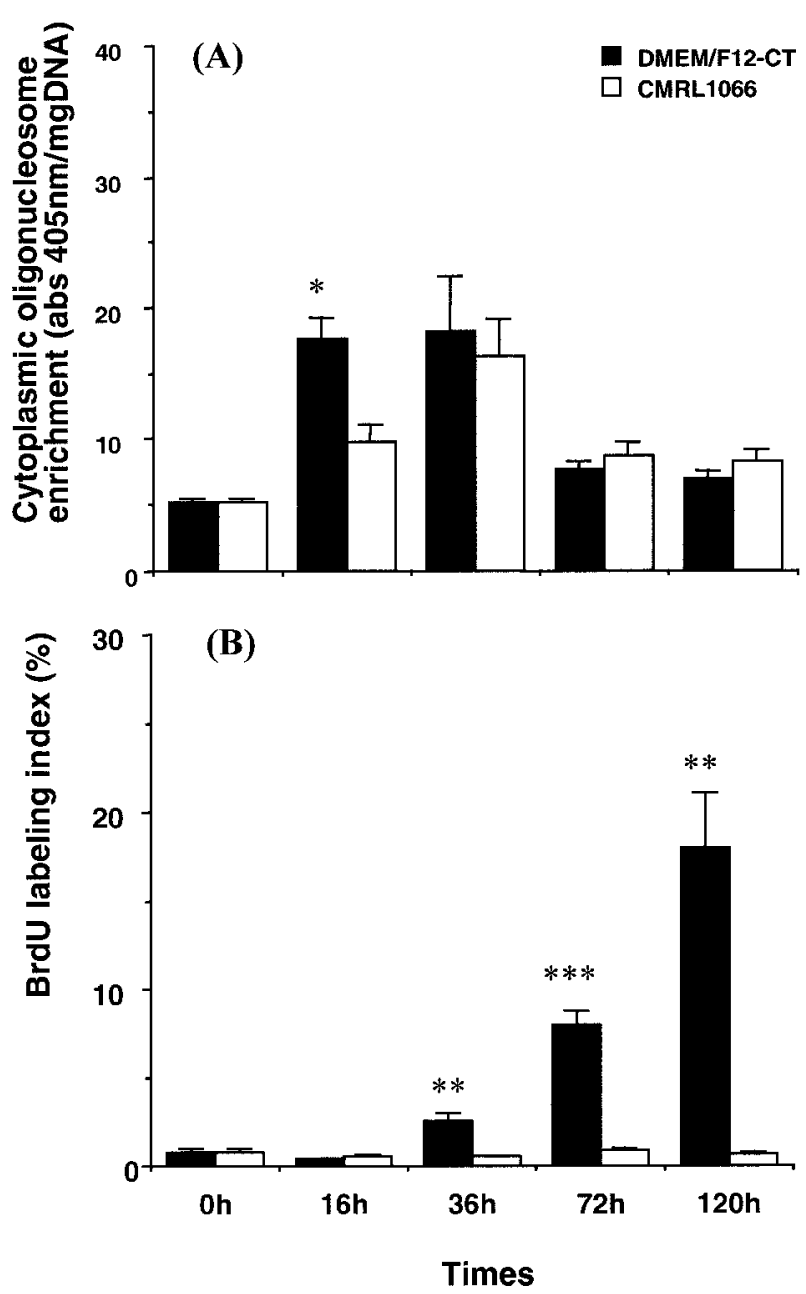

Figure 5 (A) Apoptotic activity determined by a specific cell death ELISA (at absorbance of $405 \mathrm{~nm}$ ) and (B) cell proliferation determined by BrdU labeling of islets cultured in DMEM/F12 plus CT and CMRL1066 over the time-course of cystic transformation. Note the shift to the left in the onset of apoptosis in islets in DMEM/F12 plus CT. ${ }^{*} P<0 \cdot 02,{ }^{*} P<0 \cdot 01,{ }^{*} * * P<0 \cdot 001$.

a necessary but not a sufficient requirement for transformation, and that the matrix must also play an important role in the process.

To determine whether it was the solid gel environment or the presence of extracellular matrix proteins alone that was necessary, islets were embedded in agarose gel, type 1 collagen gel or Matrigel. Only islets embedded in the latter two matrices underwent cystic transformation (Table 1). Furthermore, islets maintained in suspension in DMEM/ F12 plus CT supplemented with either soluble laminin or fibronectin failed to undergo ductal transformation. These experiments indicated that the process of transformation required the presence of extracellular matrix proteins presented in a solid gel environment.

To examine the role of integrin-mediated signaling in the transformation process in a more direct manner, islets were preincubated with the RGD motif-containing GRGDSP peptide prior to embedding in collagen. This reduced cystic transformation to $57 \%$ of the control DMEM/F12 plus CT group $(P<0 \cdot 001)$ at $72 \mathrm{~h}$ (Fig. 6A). The control peptide, GRGESP, had little influence on the transformation process. Pretreatment of islets with either soluble fibronectin or laminin prior to embedding decreased cystic transformation to $50 \%$ of control $(P<0 \cdot 01)$ at $72 \mathrm{~h}$ (Fig. 6B). Cystic transformation was further reduced to $33 \%$ of control when islets were preincubated with both GR GDSP and laminin $(P<0 \cdot 001$; Fig. 6C).

\section{Discussion}

Differentiated cells usually maintain their cellular specificities in the adult, where stability of cellular phenotype is related to a cell's interaction with its microenvironment. A perturbation or loss of stabilizing factors, however, may induce cells to change their commitment (Okada 1986). We have reported previously that isolated islets of Langerhans embedded in type 1 collagen gel can be induced to undergo transdifferentiation to duct-like epithelial structures (Yuan et al. 1996).

Little is currently known regarding the molecular events involved in transdifferentiation. Hence, the purpose of the present study was to characterize the factors involved in this transformation process in order to better understand the functional relationships that confer morphogenetic stability on cells in the isolated islet. Given the rather poor long-term success rate of cell-based therapies for diabetes mellitus, in particular islet transplantation (Rosenberg 1998), studies such as those described here could provide new insight into the issues surrounding the problem of graft failure.

There were two principal findings. First, we demonstrated that the process of cystic transformation requires both an elevation of intracellular cAMP and the presence of ECM proteins presented as a solid support. Second, we determined that the formation of a cystic structure from a solid islet sphere is a two-staged process that involves a wave of apoptosis of endocrine cells, followed by cell proliferation of the new duct-like cells.

Signal transduction during transdifferentiation has only recently become the subject of study; detailed information is therefore unavailable. It appears, however, that cAMPmediated information flow plays an important role (Osaka \& Sabban 1997, Ghee et al. 1998, Yarwood et al. 1998). In this study we found that elevation of intracellular cAMP was a necessary, but not a sufficient, condition for induction of islet-to-cyst transformation. However, it was not simply the peak value of the increase in intracellular cAMP that was important, rather it was the duration of the elevation that appeared to be associated with the highest frequency of duct epithelial transformation. This is in 
Table 1 The effect of extracellular matrix on islet-cystic transformation in isolated canine islets. The values are expressed as the percentage of cystic transformation \pm S.E.M.

\begin{tabular}{|c|c|c|c|c|}
\hline & Matrigel & Collagen I & Agarose & $\begin{array}{l}\text { Soluble } \\
\text { laminin/fibronectin }\end{array}$ \\
\hline \multicolumn{5}{|c|}{ Times (h) } \\
\hline 16 & $19 \pm 4 \cdot 7$ & $14 \pm 1 \cdot 4$ & - & - \\
\hline 36 & $49 \pm 3 \cdot 7$ & $35 \pm 3.9$ & - & - \\
\hline 72 & $60 \pm 3 \cdot 7$ & $42 \pm 1 \cdot 6$ & - & - \\
\hline 120 & $71 \pm 4 \cdot 5$ & $63 \pm 2 \cdot 4$ & - & - \\
\hline
\end{tabular}

aslets in suspension culture.

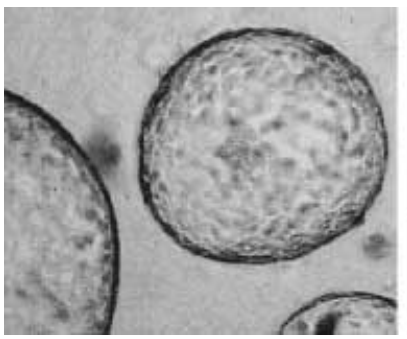

Matrigel

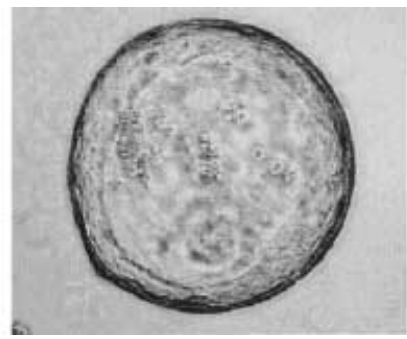

Collagen I

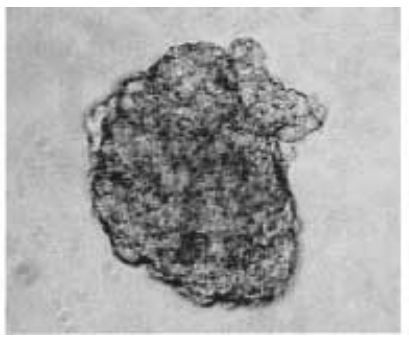

Agarose

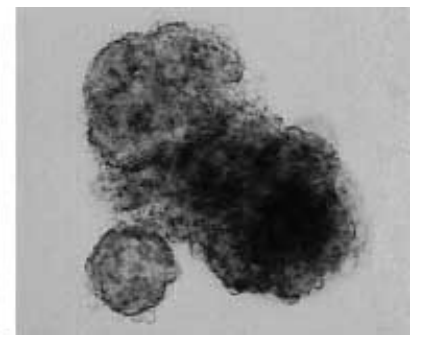

Soluble laminin keeping with Yao et al. (1995), who demonstrated the need for sustained versus transient signaling in cAMPmediated differentiation in PC12 cells. Therefore, as in other systems, the cellular responses of islet cells may be dependent on signals that elevate intracellular signals like cAMP.

An increase in intracellular cAMP is of interest too, because a rise in cAMP may form part of the effector system controlling apoptosis in pancreatic $\beta$-cells (Loweth et al. 1997). It is therefore noteworthy that cell loss due to apoptosis is the first step we observed in the process of islet-to-cyst transformation. That apoptosis should occur during islet transformation in this system is interesting, because the islets are embedded in a collagen gel, and such a matrix has been reported to help promote or maintain the differentiated state of different types of cells in culture (Rubin et al. 1981, Yang et al. 1982, Foster et al. 1983). On the other hand, extracellular matrix may also promote the process of transdifferentiation. This point is emphasized by isolated pancreatic acinar cells that transdifferentiate to duct-like structures when entrapped in Matrigel (Arias \& Bendayan 1993), and by retinal pigment epithelial cells which transdifferentiate into neurons when plated onto laminin-containing substrates (Reh et al. 1987). Most recently, Gittes et al. (1996) demonstrated, using the 11-day embryonic mouse pancreas, that the default path for growth of embryonic pancreatic epithelium is to form islets. In the presence of basement membrane constituents, however, the pancreatic anlage epithelium appears to be programmed to form ducts. This finding again emphasizes the inter-relationship between ducts and islets and highlights the important role of the extracellular matrix. Notwithstanding these observations, the presence of a solid ECM support appears to be a necessary, although not a sufficient, condition for the transformation of a solid islet to a cystic epithelial-like structure, the first stage of which involves apoptotic cell death.

Exposure to a solid matrix in the presence of CT was effective in inducing islet-to-duct transformation compared with exposure of islets to a soluble matrix protein plus CT. Since an elevation of intracellular cAMP was shown to be necessary for cystic transformation to occur, it is interesting to consider the possibility that the solid matrix contributed to the rise in cAMP and that the soluble matrix protein did not. Although we did not measure the effect of different matrix formulations on intracellular levels of cAMP, the fact that, in the absence of $\mathrm{CT}$, the solid matrix failed to induce cystic transformation suggests that it did not have any significant effect on cAMP levels. Since we found that exposure to the RGD motif reduced cystic transformation too, a similar question may be raised with respect to the effect of RGD peptide on intracellular levels of cAMP. It is well documented that RGD peptides and ECM proteins transduce their signals by interaction with the integrin receptors - with downstream signaling occurring primarily through a phosphotidylinositol-3-kinase/Akt-related pathway, and not through cAMP/protein kinase A (Matter \& Ruoslahti 2001). Even allowing for the possibility of crosstalk between these two pathways, perhaps mediated by mitogen-activated protein kinase, a control group of islets embedded into a solid matrix in the absence of CT failed to transform to ducts. This is in keeping with the report of Goberdhan et al. (1997) who showed that RGD peptide did not have an effect on intracellular cAMP in keratinocytes. 


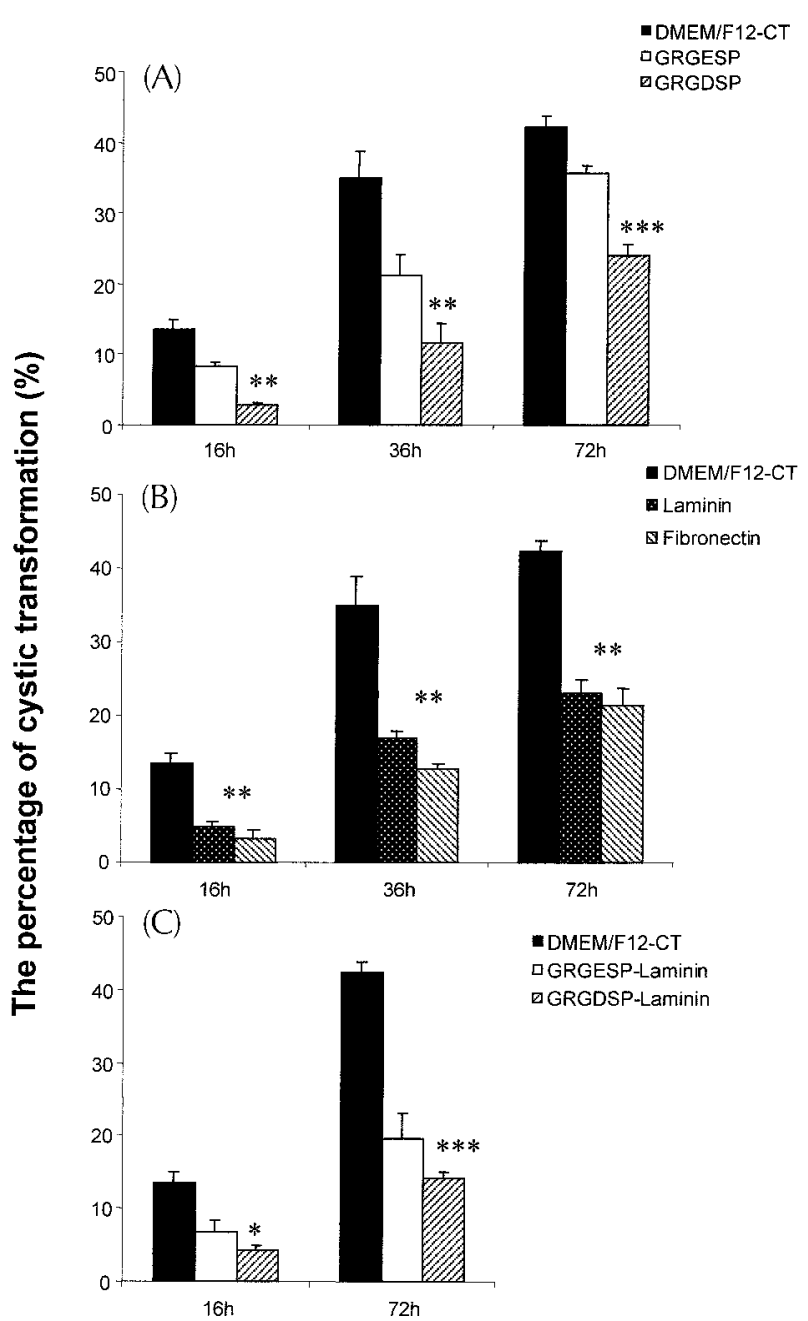

Figure 6 The effect of $(A)$ integrin-binding peptides GRGDSP and GRGESP, (B) extracellular matrix proteins laminin and fibronectin and $(C)$ a combination of GRGDSP or GRGESP and laminin on islet-cystic transformation (\%). Significant differences occurred between the GRGDSP peptide, laminin and fibronectin groups vs the control peptide, GRGESP and no treatment groups. ${ }^{*} P<0 \cdot 05$, ${ }^{* *} P<0 \cdot 01,{ }^{* *} P<0 \cdot 001$.

Conversion of a solid to a hollow structure is a morphogenetic process observed frequently during vertebrate embryogenesis (Coucouvanis \& Martin 1995). In the early mouse embryo, this process of cavitation transforms the solid embryonic ectoderm into a columnar epithelium surrounding a cavity. It has been proposed that cavitation is the result of the interplay of two signals, one from an outer layer of endoderm cells that acts over a short distance to create a cavity by inducing apoptosis of the inner ectodermal cells, and the other a rescue signal mediated by contact with the basement membrane that is required for survival of the columnar cells (Coucouvanis \& Martin 1995). The combination of these two signals results in death of inner cells not in contact with the ECM and survival of a single layer of outer cells in contact with the basement membrane. A central feature of this model is the direct initiation of apoptosis by an external signal that causes cell death. The second key feature of the model is a signal that appears to be mediated by attachment to ECM and rescues cells from cell death. There is, after all, ample precedent for cell dependence on ECM for survival (Meredith et al. 1993, Boudreau et al. 1995). In our model of islet-cystic transformation, the external death signal is probably provided by those factors that increase intracellular cAMP. Moreover, the observation that cell loss during the process of transformation occurs preferentially in the center of the islet lends support to the notion that the ECM acts as a rescue signal for those cells in the periphery. The precise role of integrins in this process remains to be more fully delineated. Integrin-ligand binding per se need not contribute to the survival signal. For example, integrins can modulate cell responsiveness to growth factors (Elliot et al. 1992).

One area not explored in the present study was the reversibility of the process of transformation. Reversibility of transdifferentiation has been reported in other cell systems (Erenpreisa \& Roach 1996). Transdifferentiation may involve cell proliferation and the appearance of a multipotential dedifferentiated intermediate cell (Yuan et al. 1996) which can express markers characteristic of several alternative phenotypes. It is possible that this is the case in our system (Yuan et al. 1996). Thus, it may be possible to expand a population of multipotential cells and then induce guided differentiation to a desired cell phenotype - in this case a mature insulin-producing $\beta$-cell. The in vitro system employed in these studies was unique for two reasons - it did not require fetal tissue, and the starting tissue, adult islets, was well defined.

In summary, this study extends our previous observation that adult islets of Langerhans can be transformed into duct epithelial cystic structures by a two-step process that involves apoptosis followed by cell differentiation and proliferation. The precise biochemical mechanism appears to involve, at least in part, elevation of intracellular cAMP, and a survival signal contributed by a solid ECM support. The differentiation potential of the cells comprising the new epithelial structure remains to be fully elucidated.

\section{Acknowledgements}

This work was supported by the Medical Research Council of Canada. R W was supported by a fellowship from the Canadian Diabetes Association in honour of Herbert L \& Francis Nussbaum. L R is a senior clinicianscientist of the Fond de la Recherche Scientfique du Quebec (FRSQ). The authors thank D Agapitos and $\mathrm{N}$ Malek for technical assistance. 


\section{References}

Arias AE \& Bendayan M 1993 Differentiation of pancreatic acinar cells into duct-like cells in vitro. Laboratory Investigation 69 518-530.

Bensley RR 1911 Studies on the pancreas of the guinea pig. American Journal of Anatomy 12 297-388.

Boudreau N, Sympson CJ, Werb Z \& Bissell MJ 1995 Suppression of ICE and apoptosis in mammary epithelial cells by the extracellular matrix. Science 267 891-893.

Coucouvanis E \& Martin GR 1995 Signals for death and survival: a two-step mechanism for cavitation in the vertebrate embryo. Cell $83279-287$.

Dudek RW \& Lawrence IE 1988 Morphologic evidence of interaction between adult ductal epithelium of pancreas and fetal foregut mesenchyme. Diabetes 37 891-900.

Ekblom P \& Timpl R 1996 Cell-to-cell contact and extracellular matrix, a multifaceted approach emerging. Current Opinion in Cell Biology 8 599-601.

Elliot B, Ostman A, Westermark B \& Rubin K 1992 Modulation of growth factor responsiveness of murine mammary carcinoma cells by cell-matrix interactions: correlation of cell proliferation and spreading. Journal of Cell Physiology 152 292-301.

Erenpreisa J \& Roach HI 1996 Epigenetic selection as a possible component of transdifferentiation. Further study of the commitment of hypertrophied chondrocytes to become osteocytes. Mechanisms of Aging and Development 287 165-182.

Foster CS, Smith CA, Dinsdale EA, Monoghan P \& Neville AM 1983 Human mammary gland morphogenesis in vitro: the growth and differentiation of normal breast epithelium in collagen gel cultures defined by electron microscopy, monoclonal antibodies and autoradiography. Developmental Biology 96 197-216.

Ghee M, Baker H, Miller JC \& Ziff EB 1998 AP-1, CREB and CBP transcription factors differentially regulate the tyrosine hydroxylase gene. Molecular Brain Research 55 101-114.

Githens S 1986 Differentiation and development of exocrine pancreas in animals. In The Exocrine Pancreas: Biology, Pathobiology and Diseases, p 21-32. Eds VLW Go, JD Gardener, FP Brooks, E Lebenthal, EP DiMagno \& GA Scheek. New York: Raven Press.

Gittes GK, Galante PE, Hanahan D, Rutter WJ \& Debas HT 1996 Lineage-specific morphogenesis in the developing pancreas - role of mesenchymal factors. Development 122 439-447.

Goberdhan NJ, Edgecombe M, Freedlander E \& MacNeil S 1997 Extracellular matrix proteins induce changes in intracellular calcium and cyclic AMP signalling systems in cultured human keratinocytes. Burns 23 122-130.

Golosow N \& Grobstein C 1962 Epithelio-mesenchymal interaction in pancreatic morphogenesis. Developmental Biology 4 242-255.

Horaguchi A \& Merrell RC 1981 Preparation of viable islet cells from dogs by a new method. Diabetes $30455-461$.

London NJM, James RFL \& Bell PRF 1992 Islet purification. In Pancreatic Islet Cell Transplantation, pp 113-123. Ed. C Ricordi. Austin: RG Landes Co.

Loweth AC, Williams GT, Scarpello JH \& Morgan NG 1997 Evidence for the involvement of cGMP and protein kinase $G$ in nitric oxide-induced apoptosis in the pancreatic B-cell line, HIT-T15. FEBS Letters 400 285-288.

Matter ML \& Ruoslahti E 2001 A signaling pathway from the alpha5 beta1 and alpha 5 beta 3 integrins that elevates bcl-2 transcription. Journal of Biological Chemistry 276 27757-27763.

Meredith JE, Fazeli B \& Schwartz MA 1993 The extracellular matrix as a cell survival factor. Molecular Biology of the Cell 4 953-961.

Okada TS 1986 Transdifferentiation in animal cells: fact or artifact? Development Growth and Differentiation 28 213-221.

Orci L, Stefan Y \& Malaisse-Lagae F 1979 Instability of pancreatic endocrine cell population throughout life. Lancet i 615.

Osaka H \& Sabban EL 1997 Requirement for cAMP/calcium responsive element but not AP-1 site in fibroblast growth factor-2elicited activation of tyrosine hydroxylase gene expression in PC12 cells. Molecular Brain Research 49 222-228.

Paraskevas S, Aikin R, Maysinger D, Lakey JRT, Cavanagh T, Wang RN \& Rosenberg L 2001 Modulation of JNK and p38 expression and activation by insulin and PD169316 in isolated islets of Langerhans. Annals of Surgery 233 124-133.

Reh TA, Nagy T \& Gretton H 1987 Retinal pigmented epithelial cells induced to transdifferentiate to neurons by laminin. Nature $\mathbf{3 3 0}$ 68-71.

Ricordi C 1992 The automated method for islet isolation. In Pancreatic Islet Cell Transplantation, pp 99-112. Ed. C Ricordi. Austin: RG Landes Co.

Rosenberg L 1998 Clinical islet transplantation. International Journal of Pancreatology 24 145-168.

Rubin K, Hook M, Obuink B \& Timpl R 1981 Substrate adhesion of rat hepatocytes: mechanism of attachment to collagen substrates. Cell 24 463-470.

Wang RN \& Rosenberg L 1999 Maintenance of beta-cell function and survival following islet isolation requires re-establishment of the islet-matrix relationship. Journal of Endocrinology 163 181-190.

Wang RN, Bouwens L \& Klöppel G 1994 Beta-cell proliferation in normal and streptozotocin treated newborn rats: site, dynamics and capacity. Diabetologia 37 1088-1096.

Yang J, Larson L, Flynn D, Elias J \& Nandi S 1982 Serum-free primary culture of human normal mammary epithelial cells in collagen gel matrix. Cell Biology International 6 969-975.

Yao H, Labudda K, Rim C, Capodieci P, Loda M \& Stork PJ 1995 Cyclic adenosine monophosphate can convert epidermal growth factor into a differentiating factor in neuronal cells. Journal of Biological Chemistry 270 20748-20753.

Yarwood SJ, Kilgour E \& Anderson NG 1998 Cyclic AMP potentiates growth hormone-dependent differentiation of 3T3-F442A preadipocytes: possible involvement of the transcription factor CREB. Molecular and Cellular Endocrinology 138 41-50.

Yuan S, Rosenberg L, Paraskevas S, Agapitos D \& Duguid WP 1996 Transdifferentiation of human islets to pancreatic ductal cells in collagen matrix culture. Differentiation 61 67-75.

Received 27 June 2001

Accepted 13 July 2001 